\title{
Una Propuesta de Evaluación Formativa en Ciencias Morfológicas
}

\author{
A Proposal of Formative Assessment in Morphology Sciences
}

Teruel, M."; Felipe, A..; Herrera, M.*; Dopazo, J."; Díaz, M."; Gómez, S."; Eyheramendy, V.* \& Guerrero, M.*

TERUEL, M.; FELIPE, A.; HERRERA, M.; DOPAZO, J.; DÍAZ, M., GÓMEZ, S.; EYHERAMENDY, V. \& GUERRERO, M. Una propuesta de evaluación formativa en ciencias morfológicas. Int. J. Morphol., 32(4):1207-1211, 2014.

RESUMEN: La carrera de Medicina Veterinaria de la Universidad Nacional del Centro de la Provincia de Buenos Aires (UNCPBA) presenta un sistema de correlatividades estricto que conlleva a frecuentes retrasos académicos. El porcentaje de alumnos que aprobó la cursada 2009 (considerando la prueba parcial y sus instancias de recuperación) del curso Histología Embriología y Teratología fue menor al $80 \%$ con una tendencia descendente considerando el periodo 2006-2009. El objetivo del trabajo fue analizar si la implementación de cambios metodológicos que incluyeran actividades con principios de evaluación formativa, lograba mejorar la calidad del proceso enseñanza-aprendizaje. La propuesta incluyó: dictado de temas evaluados en los trabajos prácticos (TP) con la antelación suficiente para permitir su lectura y comprensión; archivos power point con imágenes de las preparaciones histológicas en cada TP; evaluación de TP con devolución escrita de las correcciones; posibilidad de recuperación de los TP correspondientes a tejidos básicos contra entrega de la evaluación corregida; seminarios obligatorios y consultas concebidas como encuentros de aprendizaje previo a las mesas de exámenes finales. Como indicadores de impacto de la propuesta, se utilizaron el porcentaje de alumnos que aprobó la cursada (\%AAC) y el número de veces que rindieron el examen final hasta aprobarlo. Se compararon los periodos 2010-2012 (nueva propuesta) y 2006-2009 (cursadas tradicionales) mediante Chi2. El \%AAC fue mayor para el periodo 2010-2012 (93,1\%) comparado con el periodo 2006-2009 (82,4\%; $\mathrm{p}<0,05)$. Sin embargo, no hubo diferencias significativas en el porcentaje de alumnos que rindió entre 1 y 4 veces el examen final $(50,2 \%, 27,6 \%, 15,6 \%$ y $6,6 \%$ respectivamente; $p>0,05)$. Las modificaciones propuestas resultarían una estrategia que mejora el rendimiento académico evidenciado por un aumento en el porcentaje de aprobación de cursada, sin embargo no incidió positivamente en el desempeño académico en instancias de examen final sugiriendo la necesidad de buscar estrategias para hacer más eficiente la acreditación final del curso.

PALABRAS CLAVE: Evaluación Formativa; Enseñanza; Aprendizaje; Desempeño Académico; Ciencias Morfológicas.

\section{INTRODUCCIÓN}

Los cursos de los primeros años de las Carreras universitarias, no sólo tienen la función tradicional de impartir conocimientos disciplinares, sino además generar estrategias de orientación, apoyo y seguimiento para los estudiantes, en pos de promover el diseño y la puesta en práctica de proyectos de mejoramiento de los procesos de aprendizaje (Field et al., 2007; Rizzuto, 2009). La formación de individuos capaces de descubrir, enfrentar y solucionar problemáticas o situaciones que cotidianamente se les presentan, es fundamental para lograr no sólo la adquisición de contenidos específicos propios de cada currículo sino también aquellos aspectos que hacen a su formación profesional (Irigoin \& Vargas, 2002).

La experiencia que se describe en este trabajo, se llevó a cabo en el curso Histología Embriología y Teratología que se dicta durante el segundo cuatrimestre académico del primer año de la carrera de Medicina Veterinaria de la Universidad Nacional del Centro de la Provincia de Buenos Aires (UNCPBA).

Al analizar la trayectoria de aprobación del curso (considerando la prueba parcial y sus dos instancias de recuperación), se observó que en el año 2009 fue menor al $80 \%$ con una tendencia descendente considerando el periodo 2006-2009 (Teruel et al., 2012). Las dificultades diagnosticadas, a partir de las evaluaciones de trabajos prácticos, parciales y finales, que pudieron conducir a esos resultados se centraron en: la no adquisición y utilización adecuada de la terminología científica; la falta de valorización de las imágenes como fuente de información, atribuyéndoles sólo una función ilustrativa; la memorización de textos y

\footnotetext{
* Área de Ciencias Morfológicas, Facultad de Ciencias Veterinarias, Universidad Nacional del Centro de la Provincia de Buenos Aires (UNCPBA), Tandil, Argentina.
} 
términos sin comprender su significado; la posibilidad de elaborar representaciones gráficas correctas y ubicar referencias en ellas pero no generar proposiciones adecuadas a partir de las mismas o bien generar proposiciones adecuadas pero no poder elaborar representaciones gráficas coherentes con las mismas; la disociación de los contenidos según niveles de organización y la concepción de las disciplinas como entes aislados. Se observó también que la mayoría de los estudiantes desconocía los procedimientos para acceder a los contenidos. Este conjunto de dificultades se asoció con problemas de motivación. En este sentido se asumió que la motivación genera en los alumnos predisposición hacia los aprendizajes incidiendo a su vez en sus actitudes y, en parte, en el desempeño académico consecuente (Beguet et al., 2001). Por ello fue necesario ejercer acciones concretas, no sólo generando una actitud favorable hacia el aprendizaje de contenidos determinados, sino despertando y afianzando en los estudiantes motivos duraderos, formando intereses y valores, para que ellos mismos generaran acciones que facilitaran nuevos aprendizajes (Benítez et al., 2002). En consecuencia, se revisaron y reformularon las estrategias de enseñanza incluyendo cambios metodológicos tendientes a adaptar la formación a las posibilidades de los alumnos, a corregir los errores y a controlar la evolución de su aprendizaje basándonos en nuestros resultados (Teruel et al., 2012, 2013) y en experiencias de evaluación formativa que han logrado mejorar los resultados de aprendizaje (Grau et al., 2011).

Este trabajo tuvo como objetivo analizar si la implementación de cambios metodológicos orientados al desarrollo de actividades que incluyeran principios de evaluación formativa, lograba mejorar la calidad de los procesos de enseñanza y de aprendizaje en el curso de Histología Embriología y Teratología considerando a la evaluación como una ocasión más de aprendizaje y que en su intención y en su función formativa es más que una calificación.

\section{MATERIAL Y MÉTODO}

El curso tiene una duración de 14 semanas dentro de las cuales se desarrollan clases teóricas, trabajos prácticos, seminarios y un parcial con su correspondiente instancia de recuperación. La experiencia fue implementada tanto por los profesores como por los auxiliares de docencia y se llevó a cabo con la totalidad de los estudiantes que conformaron cada cohorte correspondiente a los años 2006 a 2012 con un valor promedio \pm desvío estándar de $178 \pm 21$ alumnos por cohorte. Se trabajó con una relación docente/alumno considerada como suficiente (1/25), de modo que esta variable no constituyera un obstáculo para el desarrollo de las actividades.
Los cambios propuestos y aplicados en el periodo 2010-2012, se centraron en modificar las diferentes actividades académicas del Curso, aportando elementos que las transformaran en situaciones percibidas por los estudiantes como instancias de aprendizaje activo, cuyas evaluaciones fueran continuas y de índole formativa. Para ello:

a) Se desarrollaron encuentros teóricos de los temas a ser evaluados en los trabajos prácticos con la antelación suficiente (5 días) como para permitir la lectura comprensiva de la bibliografía y se organizaron horarios diarios de consulta. En cada teórico se presentaron y analizaron los conceptos generales de cada unidad de contenidos y se brindaron herramientas para su estudio y para la comprensión de la lógica de las disciplinas del Curso.

b) Se incorporó en cada trabajo práctico un archivo en power point con imágenes de preparaciones histológicas que se observaban al microscopio, además de una guía con actividades de resolución individual y grupal.

c) Se evaluaron los trabajos prácticos con preguntas a desarrollar y devolución escrita de las correcciones realizadas. Las evaluaciones se orientaron a la elaboración de descripciones, el uso y asociación de términos, la búsqueda de semejanzas y diferencias, la inclusión de un concepto en uno más abarcativo, la elaboración de representaciones gráficas y el uso de referencias, la descripción de las mismas y la asociación de varias representaciones proposicionales.

d) Se estableció la posibilidad de recuperación, mediante autocorrección, de los primeros cuatro trabajos prácticos desarrollados en el curso (correspondientes a tejidos básicos), en el trabajo práctico inmediato posterior. Los estudiantes recibían de manera personalizada la devolución de sus evaluaciones con un registro por escrito de errores a manera de preguntas o comentarios breves. Se utilizaba el tiempo de las devoluciones para que cada estudiante pudiera identificar sus dificultades en el acceso a y el manejo de los contenidos.

e) Se incorporaron cuatro seminarios obligatorios donde los estudiantes trabajaron en grupos con una guía complementaria de orientación basada en preguntas de respuesta abierta, elaboración e interpretación de esquemas y realización de una exposición final con discusión de los resultados.

f) En todos los encuentros se dialogó sobre los criterios de evaluación para las instancias de pruebas parcial y final y la importancia de comprender que el Curso 
no era sólo el contenido sino las formas de acceder a él y poder utilizarlo adecuadamente. Se trabajó el "saber cómo estudiar" y la relevancia de la actitud, la predisposición, el esfuerzo, las horas dedicadas y la importancia del estudio cotidiano desde el inicio del Curso en pos de un aprendizaje construido en tiempos lógicos. Se promovió la elaboración de esquemas, el ejercicio de formular preguntas y respuestas por escrito, la autoevaluación para lograr coherencia entre lo que entienden y lo que escriben, la búsqueda concreta de material que los oriente cuando no entienden algo y la prueba de estrategias propias de estudio.

g) Previo a cada mesa de exámenes finales se programaron consultas diarias concebidas como encuentros de aprendizaje. Los alumnos debían asistir, en forma individual o grupal, con todos los materiales de estudio, sus dudas y respuestas alternativas.

Los cambios propuestos implicaron modificaciones operativas y actitudinales en los docentes, tendientes a construir un ambiente de aprendizaje donde la enseñanza se compartía con los estudiantes y se promovía el “aprender a aprender" (Grau \& Gómez, 2010).

Como indicadores de impacto de los cambios propuestos se utilizaron el porcentaje de alumnos que aprobó la cursada (\%AAC) considerando la prueba parcial y sus dos instancias de recuperación y el número de veces que cada estudiante rindió el examen final hasta aprobarlo (entre 1 y 4, máximo según Reglamento de Enseñanza y Promoción vigente en la Facultad de Ciencias Veteri- narias de la UNCPBA). Se compararon los resultados de las cohortes 2010-2012 (nueva propuesta) con los de las cohortes 2006 a 2009 utilizando prueba de Chi2.

\section{RESULTADOS}

La reformulación de estrategias de enseñanza utilizadas a partir del año 2010 permitió mejorar la calidad de los procesos de enseñanza y aprendizaje. Analizando las cohortes en conjunto, se observó que para las cohortes 20102012, el porcentaje de aprobación de la cursada, tuvo un valor medio de $93,1 \%$, resultando significativamente superior al porcentaje medio obtenido durante el periodo 20062009 que fue $82,4 \%$ ( $p<0,05)$. Paralelamente, es importante observar que el análisis del rendimiento académico tomando cada cohorte en forma individual mostró una disminución en los años 2008 y 2009 comparado con aquel de las cohortes 2006 y 2007 (Tabla I), resultados que confirman la tendencia a una disminución en el rendimiento académico de los estudiantes de la Carrera.

Los cambios propuestos incrementaron significativamente el porcentaje de alumnos que aprobó la cursada ( $\mathrm{p}<0,05$; Tabla I).

Con respecto al porcentaje de alumnos que rindió entre 1 y 4 veces el examen final, no se observaron diferencias significativas entre cohortes 2006-2009 y 2010-2012 con valores medios de 50,2\%, 27,6 \%, 15,6\% y $6,6 \%$ respectivamente ( $p>0,05$; Fig. 1$)$.

Tabla I. Porcentaje de alumnos que aprobó la cursada de Histología Embriología y Teratología según el modelo de cursada*.

\begin{tabular}{lcccc}
\hline Cohorte & $\begin{array}{c}\text { Modelo de } \\
\text { cursada }\end{array}$ & $\begin{array}{c}\text { Alumnos que } \\
\text { cursaron (n) }\end{array}$ & $\begin{array}{c}\text { Alumnos que } \\
\text { aprobaron la } \\
\text { cursada (n) }\end{array}$ & $\begin{array}{c}\text { Alumnos que } \\
\text { aprobaron la } \\
\text { cursada (\%) }\end{array}$ \\
\hline 2006 & $* *$ Tradicional & 192 & 163 & $84,89 \mathrm{ab}$ \\
2007 & Tradicional & 211 & 185 & $87,67 \mathrm{a}$ \\
2008 & Tradicional & 155 & 121 & $78,06 \mathrm{~b}$ \\
2009 & Tradicional & 194 & 153 & $78,86 \mathrm{~b}$ \\
2010 & Con cambios & 167 & 158 & $94,61 \mathrm{c}$ \\
2011 & Con cambios & 163 & 154 & $94,47 \mathrm{c}$ \\
2012 & Con cambios & 162 & 146 & $90,12 \mathrm{ac}$ \\
\hline
\end{tabular}

*Considerando la prueba parcial y sus dos instancias de recuperación.

**Tradicional: corresponde a las cohortes 2006-2009. En los trabajos prácticos no se mostraban imágenes en power point de las preparaciones observadas; las evaluaciones se realizaban sin devolución escrita de las correcciones realizadas; no se recuperaban los trabajos prácticos correspondientes a tejidos básicos en caso de resultar desaprobados; no se desarrollaban seminarios obligatorios; el diálogo docente-alumno era más ocasional o informal y no una estrategia como parte del proceso de enseñanza aprendizaje tanto durante el desarrollo del curso como previo a las mesas de exámenes finales.

Letras diferentes indican diferencias significativas $(\mathrm{p}<0,05)$. 


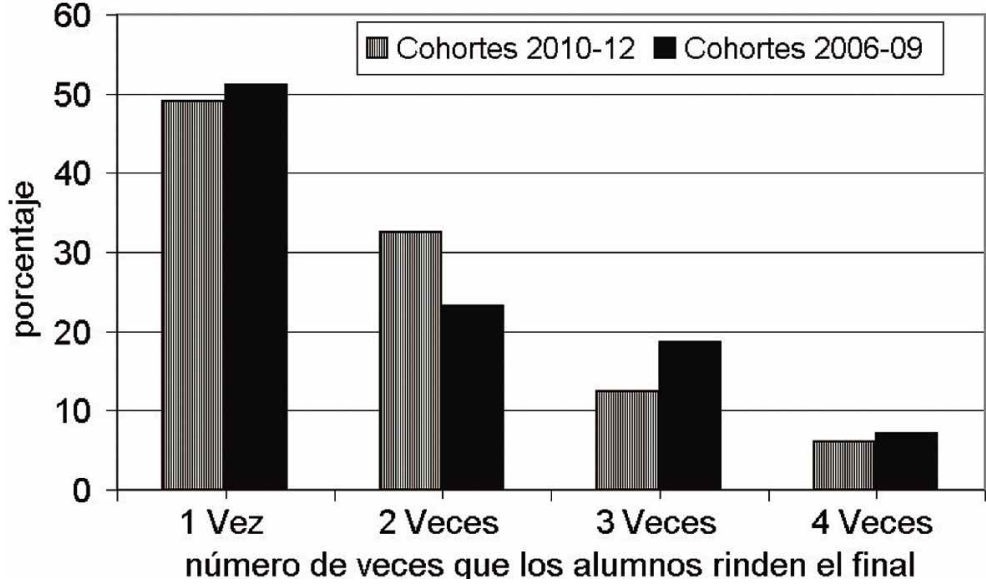

Fig. 1. Porcentaje de alumnos que rindió entre 1 y 4 veces el examen final del Curso Histología Embriología y Teratología hasta lograr su aprobación.

\section{DISCUSIÓN}

Los resultados obtenidos luego de reformular nuestras estrategias metodológicas demostraron que la utilización de evaluaciones de carácter formativo, redunda en un mejor rendimiento académico utilizándose como indicador de impacto, el porcentaje de alumnos que aprobaron la cursada. El hecho de haber planteado los criterios de evaluación en forma reiterada a lo largo del curso y hacer que los estudiantes comprendieran que el curso no era sólo el contenido sino las formas de acceder a él y poder aplicarlo, hicieron que la actitud de los mismos fuera más participativa y comprometida.

Alonso (2005), sostiene que la motivación se asocia con que las metas del aprendizaje evocan diferentes escenarios mentales en los alumnos que hacen que tengan actitudes positivas o negativas hacia el estudio lo que determina el esfuerzo que se pone para aprender y el rendimiento académico consecuente.

Diagnosticar las cuestiones problemáticas que influyen negativamente en el desempeño académico es la primera tarea que nos incumbe como docentes universitarios para luego generar estrategias de cambio o reformular las existentes. Las modificaciones introducidas en el Curso Histología Embriología y Teratología parecieran, a juzgar por los resultados que aquí presentamos, que lograron incrementar el compromiso personal de los estudiantes por aprender. Esto se tradujo en el mejor rendimiento académico observado, sugiriendo que las prácticas de elaboración de conceptos y del lenguaje para comunicarlos aunadas con un mayor vínculo docente-alumno, conformarían actividades eficaces para la mejora de los aprendizajes. de la Torre y Violant (2003), destacan la necesidad de pasar de una enseñanza basada en la información del profesor a una enseñanza basada en la actividad formativa de los estudiantes. De esta manera, como se promovió en este trabajo, los estudiantes asumen un mayor protagonismo en los procesos de enseñanza y de aprendizaje que con las metodologías tradicionales. Así, además de ir construyendo conocimientos, adquieren y/o enriquecen habilidades mediante su trabajo con orientaciones de los docentes. El ambiente del aula tiende a generar instancias de aprendizaje más implicativo y por lo tanto, resultaría más atrayente y motivador. Para los estudiantes no es suficiente el registro de la información que se les brinda, sino que resulta necesario su reelaboración y contrastación lo que permitiría lograr, como señala de la Torre (1993), "un conocimiento construido con la implicación activa del sujeto".

Sin embargo, es importante considerar que si bien el hecho de optimizar las relaciones entre los procesos de transmisión y adquisición de conocimientos con los de evaluación de carácter formativo, resultó una herramienta positiva para la aprobación de la cursada, no incidió positivamente en el desempeño académico en instancias de examen final, considerando el número de veces que los alumnos deben rendirlo hasta lograr su aprobación.

Deberán aplicarse estrategias para hacer más eficiente la acreditación final del Curso (no sólo en lo cuantitativo sino también en la evolución que el alumno ha mostrado), buscando un nexo más estrecho entre la formación continua propuesta durante el desarrollo del Curso y la evaluación final, que nos permita analizar capacidades y no sólo saberes. La evaluación formativa nos permitirá constatar los cambios logrados en los estudiantes así como la eficacia de los métodos y recursos empleados en pos de reorientar y mejorar el proceso de enseñanza-aprendizaje.

TERUEL, M.; FELIPE, A.; HERRERA, M.; DOPAZO, J.; DÍAZ, M., GÓMEZ, S.; EYHERAMENDY, V. \& GUERRERO, M. A proposal of formative assessment in morphology sciences. Int. J. Morphol., 32(4):1207-1211, 2014.

SUMMARY: The program of the UNCPBA Veterinary Medicine degree has a strict subject correlative order system leading to frequent academic delays. The percentage of students who completed the Histology Embryology and Teratology 2009 course (considering the partial tests and two resist examinations) was below $80 \%$ with a downward trend for the period 2006-2009. The objective of this work was to examine whether the implementation 
of methodological changes that include activities with principles of formative assessment could improve the quality of the teachinglearning process. The proposal included: to teach the topics evaluated in practical works (PW) allowing enough time for reading and comprehension; to present the students with power point files with images of histological preparations in each PW; to give them the PW assessment in writing; to allow resist of PWs corresponding to basic tissues after delivery of the corrected assessment; mandatory seminars and consultation meetings designed as learning instances prior to final exams. As indicators of the impact of the proposal the percentage of students who completed the course (PSC) and the number of times students sat for the final exam to pass it were taken into account. Periods 2010-2012 (new proposal) and 2006-2009 (traditional course) were compared by Chi2. The PSC was higher for the period 2010-2012 (93.1\%) compared to the 2006-2009 period (82.4\%; $\mathrm{p}<0.05)$. However, there were no significant differences in the percentage of students who sat between 1 and 4 times the final examination $(50.2 \%, 27.6 \%, 15.6 \%$ and $6.6 \%$ respectively, $\mathrm{p}>0.05)$. The changes proposed would become a strategy to improve the academic performance as evidenced by an increase in the percentage of students completing the course; however, they had no positive impact on the academic performance at the final examinations instances suggesting the need to find strategies to make more efficient the final accreditation of the course.

KEY WORDS: Formative assessment; Teaching; Learning; Academic performance; Morphology sciences.

\section{REFERENCIAS BIBLIOGRÁFICAS}

Alonso, J. Motivaciones, expectativas y valores-intereses relacionados con el aprendizaje: el cuestionario MEVA. Psicothema [en línea], 17(3):404-11, 2005.

Beguet, B.; Cortada de Kohan, N.; Castro Solano, A. \& Renault, G. Factores que intervienen en el rendimiento académico de los estudiantes de psicología y psicopedagogía. Evalu-Acción: Revista Científica de la Dirección de Evaluación y Acreditación de la Secretaría General de la Universidad del SalvadorUSAL [en línea], 1(1), 2001. Disponible en: http:// www.salvador.edu.ar/uc4-pub-01-1-1-04.htm

Benítez, M. E.; Jiménez, M. C.; Osicka, R. M. \& Moro, L. A. ¿Es necesario motivar el aprendizaje en la universidad?. Una experiencia en Química Analítica General. Corrientes, Universidad Nacional del Noreste, Argentina - Facultad de Agroindustrias - Cátedra de Química Analítica General, 2002. Disponible en: http://www.unne.edu.ar/unnevieja/Web/cyt/cyt/ 2002/09-Educacion/D-023.pdf

de la Torre, S. La creatividad en la aplicación del método didáctico. En: Sevillano, M. L. Estrategias metodológicas en la formación del profesorado. Madrid, UNED, 1993. pp.287-309. de la Torre, S. \& Violant, V. Estrategias creativas en la enseñanza universitaria. Creatividad y Sociedad [en línea], (3), 2003. Disponible en: http://www.ub.edu/sentipensar/pdf/saturnino/ estrategias_creativas_universitaria.pdf

Field, S.; Kuczera, M. \& Pont, B. No More Failures: Ten Steps to Equity in Education. Paris, OECD, 2007.

Grau, S.; Alvarez, J. \& Tortosa Ibáñez, M. Una estrategia innovadora en la docencia universitaria: la evaluación formativa. En: Roig Vila, R. \& Laneve, C. (Eds.). La práctica educativa en la sociedad de la información. Innovación a través de la investigación. Alcoy-Brescia, Marfil \& La Scuola Editrice, 2011. pp.175-85.

Grau, S. \& Gómez, M. C. La evaluación, un proceso de cambio para el aprendizaje. En: Gómez, M. C. \& Grau, S. (Eds.). Evaluación de los aprendizajes en el Espacio Europeo de Educación Superior. Alcoy, Marfil, 2010. pp.17-33.

Irigoin, M. \& Vargas, F. Competencia Laboral: Manual de conceptos, métodos y aplicaciones en el sector salud. Montevideo, OPS-CINTERFOR, 2002.

Teruel, M.; Felipe, A.; Herrera, M.; Díaz, M.; Dopazo, J.; Eyheramendy, V. \& Gómez, S. Alternativas para mejorar la calidad de la enseñanza-aprendizaje de los alumnos del curso Histología Embriología Teratología. La Plata, XIV Congreso de Ciencias Morfológicas y 11avas Jornadas de Educación. Sociedad de Ciencias Morfológicas de La Plata, Argentina, 2012.

Teruel, M.; Felipe, A. \& Herrera, M. Experiencias de evaluación formativa en Ciencias Morfológicas. V Encuentro Nacional y II Latinoamericano sobre Ingreso a la Universidad Pública. Políticas y estrategias para la inclusión. Nuevas complejidades; nuevas respuestas. Universidad Nacional de Luján, Argentina, 2013.

Rizzuto, F. La deserción en la educación superior, motivos y medidas preventivas. Tesis de Licenciatura en Organización y Gestión Educativa, Escuela de Educación, Universidad Austral, Argentina, 2009.

Dirección para Correspondencia: Miriam Teruel, Med Vet. MSc.

Prof. Histología Embriología y Teratología

Facultad de Ciencias Veterinarias

Universidad Nacional del Centro de la Provincia de Buenos

Aires

UNCPBA Tandil, Provincia de Buenos Aires

ARGENTINA

Email: mteruel@vet.unicen.edu.ar

Recibido : 14-04-2014 Aceptado: 21-08-2014 